\title{
Potential Antithrombotic Effects of Bupivacaine in Malignancies: An In Vitro Assesment using Thromboelastography
}

B. A. BILIR, M. O. AKAY ${ }^{1}$, D. CEYHAN* AND F. S. MUTLU² 
Department of Anesthesiology and Reanimation, ${ }^{1}$ Department of Hematology, ${ }^{2}$ Department of Biostatistics, Eskişehir Osmangazi University Medical School, Eskisehir, Turkey

\title{
Bilir, et al.: Potential Antithrombotic Effects of Bupivacaine
}

\begin{abstract}
Thrombosis is a serious complication in patients with gynecologic malignancy. Bupivacaine may have potential beneficial effects on hypercoagulable state in patients with gynecologic malignancies when used either for postoperative analgesia or for intractable pain in terminal cancer patients. The purpose of this study was to investigate the direct effects of bupivacaine in women with gynecologic malignancies. A total of 22 patients with gynecological tumors were included in the study. Thromboelastography analysis was performed with the rotational thromboelastometry for the whole blood samples. Clotting time, clot formation time and maximum clot formation were recorded. In INTEM assay, clotting time and clot formation time values were significantly prolonged with the addition of bupivacaine compared with the non-medicated samples $(\mathbf{P}<\mathbf{0 . 0 5})$. In comparison with the non-medicated samples, there was a similar significant increase in clotting time, clot formation time values and a decrease in maximum clot formation value in EXTEM assay in the bupivacaine incubated samples $(\mathrm{P}<0.05)$. The results suggest that bupivacaine causes hypocoagulable changes on the thromboelastogram profile of gynecologic cancer patients, in vitro.
\end{abstract}

Key words: Bupivacaine, coagulation, thromboembolism, cancer, thromboelastography

The association between malignancy and thrombosis has been reported in many studies ${ }^{[1,2]}$. Cancer cells activate the hemostatic system by producing molecules with procoagulant, fibrinolytic or proaggregating activities. Procoagulant effects are considered to play main role in the development of a tendency to hypercoagulable status in cancer. Also, cancer cells have indirect effects in release of proinflammatory cytokines and through interactions between tumor cells and host vascular cells ${ }^{[1]}$.

Hypercoagulability, is a term used to describe an increased risk of thrombosis due to genetic defects or acquired coagulation or non-coagulant factor, is hard to diagnose by standard laboratory tests unless marked increases occur in platelet count and fibrinogen concentrations in cancer patients ${ }^{[1]}$. As clotting is a dynamic process, a global assessment of hemostatic function would be more satisfactory in evaluating the changes of the coagulation status in cancer. Thromboelastography (TEG) is a sensitive method that is able to diagnose hypercoagulability which is not detected by routine coagulation tests ${ }^{[1]}$. It differs from other laboratory coagulation tests by measuring the clotting process from the time when fibrin strands are formed to fibrinolysis ${ }^{[3]}$. It is used to rapidly assess the interaction of platelets with the protein coagulation cascade from initial platelet-fibrin interaction, through platelet aggregation, clot strengthening, and fibrin crosslinking, to eventual clot lysis.

Wehrum et $a l .{ }^{[3]}$ reported that hypercoagulability

*Address for correspondence

E-mail: drdceyhan@gmail.com

July-August 2016 is more common among women with gynecologic malignancies compared with age-matched controls and the impact of thrombosis in gynecologic cancer patients is considerable in terms of inconvenience, mortality and morbidity. On the other hand, these patients usually need major surgical procedures and surgery itself is an important contributing factor to hypercoagulable states. In this context, any agent that can attenuate hypercoagulable state would be of great value, particularly in those patients undergoing surgery or in patients confined to bed in terminal cancer period. Bupivacaine is a commonly used local anesthetic agent for this group of patients. We hypothesized that bupivacaine may have potential beneficial effects on hypercoagulable state in patients with gynecologic malignancies when used either for postoperative analgesia or for intractable pain in terminal cancer patients. So, the purpose of this study was to investigate the direct effects of bupivacaine on thromboelastography parameters in women with gynecologic malignancies in vitro.

A total of 22 patients with gynecological tumors were eligible for the study after obtaining approval from the University Medical Faculty Ethics Committee and written informed consent. Patients with a history of

This is an open access article distributed under terms of the Creative Commons Attribution-NonCommercial-ShareAlike 3.0 License, which allows other the remix, tweak, and build up to the non-commercially, as long as the author is credited and the new creations are licensed under the identical terms.

Accepted 14 August 2016

Revised 27 July 2016

Received 02 June 2016

Indian J Pharm Sci 2016;78(4):552-556 
hematological or coagulation disorders, those taking anticoagulant therapy and antiplatelet therapy, history of recent antiinflammatory drug or heparin (within 2 weeks of enrollment) and those with renal or liver function were excluded. Blood samples were obtained using a 19-gauge needle under minimum stasis. Blood was collected using a two-syringe technique. After discarding the initial $2 \mathrm{ml}$ of blood to prevent tissue thromboplastin contamination, samples for TEG analysis was drawn into $4.5 \mathrm{ml}$ vacutainers (Becton Dickinson) containing 3.2\% trisodium citrate with a citrate/blood ratio of 1:9.

TEG analysis was performed with the rotational thromboelastometry $\left(\right.$ ROTEM $\left.^{\circledR}\right)$ Coagulation Analyzer (Pentapharm, Munich, Germany). The method and the parameters of ROTEM have been described in detail previously by Akay et $a l^{[1]}$. The local anesthetic concentration was prepared to produce a final blood concentrations equal to $0.125 \%$ bupivacaine ${ }^{[4,5]}$. After incubating the test solution at $37^{\circ}$ for $2 \mathrm{~min}, 300 \mu 1$ citrated blood was re-calcified with $20 \mu 10.2 \mathrm{~mol} / 1$ $\mathrm{CaCl}_{2}$ (star-TEM ${ }^{\circledR}$; Pentapharm, Munich, Germany) and activation of coagulation was performed with different agents. In INTEM, coagulation is activated with $20 \mu 1$ of contact activator (partial thromboplastinphospholipid from rabbit brain extract and ellagic acid, in-TEM ${ }^{\circledR}$; Pentapharm, Munich, Germany). In EXTEM coagulation is activated by $20 \mu \mathrm{l}$ of tissue factor (TF, tissue thromboplastin from rabbit brain extract, ex-TEM ${ }^{\circledR}$; Pentapharm, Munich, Germany). All TEG samples were analyzed within 30-90 min of blood collection.

The following ROTEM parameters, clotting time (CT), clot formation time (CFT) and maximum clot formation (MCF) were recorded. Clotting time $(\mathrm{CT}=\mathrm{R})$ is the time from start of the measurement until initiation of clotting. CT is influenced by activities of coagulation factors. Clot formation time $(\mathrm{CFT}=\mathrm{k})$ is the time from initiation of clotting until a clot firmness of $20 \mathrm{~mm}$ is detected. CFT is influenced by activities of coagulation factors, platelet count/function, thrombin formation, fibrin precipitation, fibrinogen and hematocrit. Maximum clot formation $(\mathrm{MCF}=\mathrm{MA})$ represents firmness of the clot. It is affected by fibrin and fibrinogen concentration, platelet count/function, thrombin concentration, factor XIII, and hematocrit.

Statistical analysis was carried out using the SPSS 20. Normally distributed continuous dependent variables were analyzed using paired t test and presented as mean and standard deviation. Non normal distribution variables were compared with Wilcoxon test for dependent two groups and presented as median (25th to 75 th percentile). $\mathrm{P}$ value less than $0.05(\mathrm{P}<0.05)$ was accepted significant.

Twenty two patients with gynecologic malignancies in similar stages were included in the study. Results of ROTEM parameters are presented in Table 1. The results of non-medicated whole blood control ROTEM parameters were similar in all patients. In INTEM assay, CT $(\mathrm{P}<0.05)$ and $\mathrm{CFT}(\mathrm{P}<0.05)$ values were significantly increased and $\mathrm{MCF}(\mathrm{P}<0.05)$ value was significantly decreased in bupivacaine-medicated samples compared with non-medicated samples. In comparison with the non-medicated samples, there was a similar significant increase in $\mathrm{CT}(\mathrm{P}<0.05)$ and CFT $(\mathrm{P}<0.05)$ values and a decrease in $\mathrm{MCF}(\mathrm{P}<0.05)$ value in EXTEM assay in the medicated samples. Blood medicated with bupivacaine generated slow (increased CT, CFT) and weak (decreased MCF) clot. As anticipated, the ROTEM parameters of medicated blood of patients with gynecological tumors suggest a hypocoagulable state when compared with their baseline parameters.

Results of our study confirm that bupivacaine affects coagulation system in vitro. The changes in coagulation observed using the ROTEM demonstrated tendency to hypocoagulable state in women with gynecologic malignancies.

In a previous study, the hypercoagulable state of patients with gynecologic cancer has been demonstrated by $\mathrm{TEG}^{[1]}$. Common additional factors

TABLE 1: VALUES OF ROTEM® PARAMETERS IN THE NON-MEDICATED AND MEDICATED SAMPLES

\begin{tabular}{lllll}
\hline & Parameters & Non-medicated & Bupivacaine & P-value \\
\hline INTEM & CT (s) & $172.5 \pm 25.1$ & $229.7 \pm 33.7$ & $*$ \\
& CFT (s) & $65.5(52.25-77.75)$ & $178.0(163.5-213.5)$ & $*$ \\
EXTEM & MCF (mm) & $65.7 \pm 5.3$ & $46.3 \pm 6.7$ & $*$ \\
& CT (s) & $68(59.75-78.25)$ & $87.5(76.5-108.0)$ & $*$ \\
& CFT (s) & $84.5(58.75-92.75)$ & $197.5(177.5-218.75)$ & $*$ \\
\hline
\end{tabular}

INTEM, partial thromboplastin-phospholipid; EXTEM, tissue thromboplastin; CT, clotting time; CFT, clot formation time; MCF, maximum clot firmness. Data are given as mean $\pm S D$, median (25th to 75th percentile); ns means non-significant; *P-value $<0.05$ 
like limited mobilization caused by intractable pain and major surgical procedures for the tumor resection or metastases further promote this thrombotic tendency. Deep venous thrombosis, pulmonary embolism and myocardial infarction are frequently encountered thromboembolic complications after major surgery and these complications are directly related with postoperative morbidity, mortality and higher health care $\operatorname{costs}^{[6]}$. Moreover, surgery for gynecologic malignancies shows several differences from other surgical procedures. Women with cancer often require major intrapelvic procedures, such as lymph node dissection and excision of peritoneal metastases, and it is likely that thromboembolic complications will occur at a higher incidence in these patients ${ }^{[7]}$. Hypercoagulable state is initiated during the surgical procedure by the factors like tissue proteins released from damaged vessels, local tissue trauma, reduction in venous blood flow secondary to positive pressure ventilation, neuromuscular blockade, inflammatory cytokine activation and sympathetic system activation ${ }^{[6,8]}$. Sympathetic response to surgery gives rise to pronounced increases in factor VIII and factor VIII related antigen, inhibits fibrinolysis through PAI-1, decreases antithrombin ${ }^{[8]}$. All these changes in coagulation cascade initiate platelet aggregation during the surgery. These patients may be exposed to many different agents that can affect platelet function and coagulation during their therapy. Epidural local anesthetics are commonly used agents in a cancer patient undergoing major abdominal surgery or suffering from severe pain. There have been many proposed benefits of perioperative epidural usage in cancer patients after major abdominal surgery include excellent pain therapy, reduction in postoperative cardiovascular complications, pulmonary failure and venous thromboembolic events ${ }^{[9]}$. Additionally, longterm percutaneous epidural catheterization is one of the most preferred techniques for the pain relief in terminally ill gynecologic cancer patients. This method appears to be most effective pain management method in patients suffering from pain due to nerve root involvement ${ }^{[10]}$.

Epidural anesthesia and analgesia have the potential to reduce or eliminate the physiologic responses to surgery by blunting the sympathetic response and improving lower extremity blood flow. This technique attenuates the hypercoagulable perioperative state and thereby decreases thromboembolic complications and improves outcome. Moraca et al. ${ }^{[8]}$ reported that sympathetic denervation with epidural local anesthetics is associated with demonstrable effects on the coagulation cascade, by the normalization of factor VIII and factor VIII-related protein, decreases in PAI-1, and an increased antithrombin III. However, sympathetic denervation alone does not seem to be a likely explanation for the observed changes in the coagulation system. Concurrently, systemic absorption of local anesthetics may exert anticoagulant effect by blocking thromboxane A2 signaling and reduce blood viscosity by minimizing protein, erythrocyte, and platelet aggregation ${ }^{[8,11]}$. The systemic absorption of epidural local anesthetics and sympathetic denervation are likely decrease the incidence of clot formation besides the known beneficial effects like improved pain control and earlier mobilization. Since hypercoagulable state is more prevalent and more profound in women during the first $24 \mathrm{~h}^{[6,12]}$, postoperative epidural analgesia with local anesthetics may have pronounced useful effects in patients who are at risk for developing complications related to a hypercoagulable state.

Studies that investigate the direct effects of bupivacaine on coagulation had different results. Some of the studies showed that bupivacaine has no significant effects on the thromboelastographic variables in vitro ${ }^{[4,13]}$. In a study performed by Benzon et al. ${ }^{[14]}$ it is reported that there is no beneficial effect of adding bupivacaine to an epidural infusion on postoperative coagulation status. In contrast, Kohrs et al. ${ }^{[15]}$ reported that after incubation of whole blood with $2 \mu \mathrm{m}$ bupivacaine thromboelastography reaction time and coagulation growth time increases significantly and maximal amplitude decreased. In another study it is demonstrated that bupivacaine in concentration of $0.125 \%$ and $0.25 \%$ significantly impairs coagulation in a concentrationdependent manner by thromboelastography ${ }^{[5]}$. The authors of this study mentioned that besides the known neuronal action of local anesthetics by blocking membrane ion channels, they may also stabilize the membrane of platelets, inhibiting both alpha-granule release, thromboxane A2 signaling and aggregation ${ }^{[5]}$.

There have been no previous studies on the direct effects of local anesthetics on the hypercoagulable state of patients with gynecologic cancer. We aimed to determine thromboelastographic changes to observe whether bupivacaine influences the coagulation status of these patients. Bupivacaine affected the initiation and the speed of clot formation as reflected in the values of CT and CFT and also impaired clot strength as represented by lower MCF values. Our data presented a thromboelastographic evidence of bupivacaine-induced hypocoagulability in patients with gynecological tumors. It seems that systemically absorbed bupivacaine may have direct effects on 
coagulation concomitant with proved indirect effects of epidural block. To our knowledge, our findings represent the first time that the use of bupivacaine may prevent from malignancy-induced hypercoagulability and from related thromboembolic complications by direct effects on coagulation. At this point another reasonable concern arises. Do the local anaesthetics cause bleeding? Hollmann et al. ${ }^{[16]}$ elucidated this point that local anesthetics abolish hypercoagulability without effecting physiologic aggregation and coagulation processes. The use of local anesthetics does not lead to an increase in postoperative bleeding as observed with anticoagulants.

As we mentioned in our previous report, unless the pronounced increment occurs in platelet count and fibrinogen concentration, it is hard to diagnose hypercoagulable status with routine coagulation tests in cancer patients. TEG is a sensitive method that is able to identify the hemostatic state and measure hypercoagulability ${ }^{[1]}$. This technique allows rapid global assessment of hemostatic function using whole blood. In our study we prefer to perform TEG analyses with the ROTEM Coagulation Analyser because this advanced technique has avoided several limitations of classical thromboelastogram while maintaining a good correlation with conventional TEM determination ${ }^{[1]}$.

However the clinical relevance of this in vitro study has some limitations. First limitation is that it should be realized that we only studied thromboelastographic variables in cancer patients before the surgical procedure. Subsequent studies should investigate if the effect grows with concomitant surgery. Second limitation may be the incubation time. The inhibitory effect of bupivacaine may increase after longer incubation times. Since, under clinical conditions of epidural anesthesia and analgesia local anesthetics have prolonged contact with blood.

We can infer that bupivacaine affects the initiation and the speed of clot formation and also impaired clot strength. The results suggest that bupivacaine causes hypocoagulable changes on the thromboelastogram profile of gynecologic cancer patients, in vitro. Moreover, the results of our in vitro study need to be confirmed by follow up clinical studies.

\section{Financial support and sponsorship:}

Nil.

\section{Conflicts of interest:}

There are no conflicts of interest.

\section{REFERENCES}

1. Akay OM, Ustuner Z, Canturk Z, Mutlu FS, Gulbas Z. Laboratory investigation of hypercoagulability in cancer patients using rotation thrombelastography. Med Oncol 2008;26:358-64.

2. Winter PC. The pathogenesis of venous thromboembolism in cancer: emerging links with tumour biology. Hematol Oncol 2006;24:126-33.

3. Wehrum MJ, Hines JF, Hayes EB, Kost ER, Hall KL, Paidas MJ. Comparative assessment of hypercoagulability in women with and without gynecologic malignancies using the thromboelastograph coagulation analyzer. Blood Coagul Fibrinolysis 2010;21:140-43.

4. Siau C, Ng HP, Tan GM, Ho BS, Pua HL. In vitro effects of local anaesthetics on the thromboelastographic profile of parturients. Br J Anaesth 2005;94:117-20.

5. Tobias MD, Henry C, Augostides YG. Lidocaine and bupivacaine exert differential effects on whole blood coagulation. J Clin Anesth 1999;11:52-5.

6. Dai Y, Lee A, Critchley LA, White PF. Does thromboelastography predict postoperative thromboembolic events? A systematic review of the literature. Anesth Analg 2009;108:734-42.

7. Suzuki N, Yoshioka N, Ohara T, Yokomichi N, Nako T, Yahagi $\mathrm{N}$, et al. Risk factors for perioperative venous thromboembolism: A retrospective study in Japanese women with gynecologic diseases. Thromb J 2010;7:8-17.

8. Moraca RJ, Sheldon DG, Thirlby RC. The role of epidural anesthesia and analgesia in surgical practice. Ann Surg 2003;238:663-73.

9. Ferguson SE, Malhotra T, Seshan VE, Levine DA, Sonoda Y, Chi DS, et al. A prospective randomized trial comparing patient-controlled epidural analgesia to patient-controlled intravenous analgesia on postoperative pain control and recovery after major open gynecologic cancer surgery. Gynecol Oncol 2009;114:111-16.

10. Ali NM, Hanna N, Hoffman JS. Percutaneous epidural catheterization for intractable pain in terminal cancer patients. Gynecol Oncol 1989;32:22-5.

11. Borg T, Modig J. Potential antithrombotic effects of local anaesthetics by their inhibition of platelet aggregation. Acta Anaesthesiol Scand 1985;29:739-42

12. Schreiber MA, Differding J, Thorborg P, Mayberry JC, Mullins RJ. Hypercoagulability is most prevalent early after injury and in female patients. J Trauma 2005;58:475-80.

13. Gibbs NM, Sear JW. Effect of ketorolac, bupivacaine and lowdose heparin on thrombelastographic variables in vitro. Br J Anaesth 1995;75:27-30.

14. Benzon HT, Wong CA, Wong HY, Brooke C, Wade L. The effect of low-dose bupivacaine on postoperative epidural fentanyl analgesia and thrombelastography. Anesth Analg 1994;79:911-7.

15. Kohrs R, Hoenemann CW, Feirer N, Durieux ME. Bupivacaine inhibits whole blood coagulation in vitro. Reg Anesth Pain Med 1999;24:326-30.

16. Hollmann MW, Wieczorek KS, Smart M, Durieux ME. Epidural anesthesia prevents hypercoagulation in patients undergoing major orthopedic surgery. Reg Anesth Pain Med 2001;26:215-22. 